\title{
Does Combination Therapy of Popliteal Sciatic Nerve Block and Adductor Canal Block Effectively Control Early Postoperative Pain after Total Knee Arthroplasty?
}

\author{
Jin-Hyeok Seo, MD, Seung-Suk Seo, MD, Do-Hun Kim, MD, Byung-Yoon Park, MD, Chan-Ho Park, MD, and \\ Ok-Gul Kim, MD
}

Department of Orthopedic Surgery, Bumin General Hospital, Busan, Korea

\begin{abstract}
Purpose: We compared adductor canal block (ACB) alone and a combination of ACB and sciatic nerve block (SNB) to control early postoperative pain after total knee arthroplasty.

Materials and Methods: One hundred patients received continuous ACB alone (group A), and another 100 patients received continuous ACB and single popliteal SNB (group B). Pain was evaluated at rest and $45^{\circ}$ knee flexion using the numeric rating scale (NRS). The number of times the patient pressed the intravenous patient-controlled analgesia (PCA) button, total PCA volume infused, and the total dosage of additional analgesics were evaluated. We also investigated complications associated with each pain control technique.

Results: The NRS score on postoperative day 1 was significantly lower in group B than in group A. The number of times patients pressed the PCA button on postoperative day 1 and the total infused volume were significantly lower in group B than in group A. Thirty-five (35\%) patients in group B developed foot drop immediately after surgery; but they all fully recovered on postoperative day 1 .

Conclusions: SNB can be effective for management of early postoperative pain that persists even after ACB. Further research is needed to determine the proper dosage and technique for reducing the incidence of foot drop.
\end{abstract}

Keywords: Knee, Arthroplasty, Pain, Nerve block, Sciatic nerve, Adductor canal

\section{Introduction}

Postoperative pain is fairly common in patients who have undergone total knee arthroplasty (TKA). Serious pain after TKA can eventually lead to poor clinical outcomes because it can interfere with early rehabilitation and extend the length of hospital stay $^{1)}$. While a variety of techniques are used to manage postoperative pain, peripheral nerve blocks have recently gained popu-

Received April 12, 2017; Revised (1st) June 9, 2017; (2nd) July 20, 2017; Accepted August 3, 2017

Correspondence to: Ok-Gul Kim, MD

Department of Orthopedic Surgery, Bumin Hospital, 59 Mandeokdaero, Buk-gu, Busan 46555, Korea

Tel: +82-51-330-3000, Fax: +82-51-337-5041

E-mail:pnuh3@hanmail.net

This is an Open Access article distributed under the terms of the Creative Commons Attribution Non-Commercial License (http://creativecommons.org/licenses/by-nc/4.0/) which permits unrestricted non-commercial use, distribution, and reproduction in any medium, provided the original work is properly cited. larity ${ }^{2}$. In particular, femoral nerve block (FNB) is considered the gold standard. However, even after a successful FNB, about $60 \%-90 \%$ of patients still require further treatment for severe postoperative pain ${ }^{3,4)}$. The pain originates from the posterior side of the knee, which is predominately innervated by the sciatic and obturator nerves ${ }^{5)}$. Either sciatic nerve block (SNB) or an intraoperative injection to the back of the knee is used to reduce persistent posterior knee pain after $\mathrm{FNB}^{6-8)}$. Recently, the use of adductor canal block (ACB) has emerged as a promising alternative to FNB and has been shown to effectively manage postoperative pain and minimize the decrease in quadriceps muscle size ${ }^{9,10)}$.

Nonetheless, to our knowledge, no previous studies have reported the effectiveness of popliteal sciatic nerve block (PSNB) to reduce persistent postoperative pain despite $\mathrm{ACB}$. In this study, we compared the efficacy of $\mathrm{ACB}$ alone and a combination of $\mathrm{ACB}$ and PSNB to control early postoperative pain after TKA. 


\section{Materials and Methods}

This retrospective study was approved by the Institutional Review Board of our hospital (201704-BM-001). We evaluated patients who underwent TKA in our hospital's department of orthopedics between October 2015 and June 2016. Patients met criteria for inclusion if they were older than 55 years and had osteoarthritis with Kellgren and Lawrence grade III to IV and American Society of Anesthesiologists physical status classification I to III. We excluded patients with a history of surgeries (e.g., contralateral knee surgery within 3 months and spine surgery within 6 months), allergy to any local anesthetics, history of chronic pain requiring treatment with long-acting opioids, inability to understand the numeric rating scale (NRS), and contraindications to peripheral nerve block (e.g., localized infections, sepsis, or preexisting lower extremity neurological abnormality).

We compared a control group of 100 cases who received ACB alone (group A) with an experimental group of 100 cases who received $A C B$ and $\mathrm{PSNB}$ (group B).

We performed ACB to former 100 cases and then ACB and PSNB to latter 100 cases. All peripheral nerve blocks were performed before the spinal anesthesia by the same anesthesiologist who had experiences with ultrasound-guided peripheral nerve block.

The ultrasound-guided popliteal approach to SNB was performed according to the technique originally described by Sinha and $\mathrm{Chan}^{11)}$ With the patient in the prone position, an ultrasound probe was moved around the popliteal crease and block of the sciatic nerve was performed immediately proximal to its bifurcation into common peroneal and tibial nerves with $20 \mathrm{~mL}$ of $0.75 \%$ ropivacaine. The patient was then placed in the supine position to receive the ultrasound-guided ACB. We identified the femoral artery and the saphenous nerve just lateral to the artery in the medial part of the thigh, halfway between the superior anterior iliac spine and the patella. A needle was inserted and 2 to $3 \mathrm{~mL}$ of normal saline was injected to check correct placement of the needle. And then $0.75 \%$ ropivacaine $20 \mathrm{~mL}$ was injected to the adductor canal. A catheter (Perfix; B.BRAUN, Melsungen, Germany) was inserted for continuous ACB. All patients had tricompartmental cemented knee arthroplasty with PCLsubstituting implants under tourniquet control. Patients received $10 \mathrm{~mL}$ of $0.2 \%$ ropivacaine injections four times daily for three days via catheter to maintain continuous ACB after TKA. The catheter was removed after the last infusion at 6 am on day 4 postoperatively. In addition, both groups received intravenous patient-controlled analgesia (IV-PCA) to manage postoperative pain. The analgesics used for IV-PCA included fentanyl $600 \mathrm{mcg}$ $(12 \mathrm{~mL})$, ketorolac tromethamine $90 \mathrm{mg}(3 \mathrm{~mL})$, and Nepofam hydrochloride $20 \mathrm{mg}(20 \mathrm{~mL})$, which were diluted in $150 \mathrm{~mL}$ saline. The IV-PCA pump was set to deliver a loading dose of 2 $\mathrm{mL}$, a continuous infusion of $0.1 \mathrm{~mL} / \mathrm{h}$ with a lockout interval of 20 minutes, and a 1-hour dose limit of $6 \mathrm{~mL}$. The IV-PCA was removed with the ACB catheter at 6 am on postoperative day 4 . Standard oral analgesics consisted of acetaminophen $325 \mathrm{mg}$, tramadol hydrochloride $37.5 \mathrm{mg}$, and meloxicam $7.5 \mathrm{mg}$ every 12 hours. After postoperative day 1, patients were permitted ambulation with the assistance of a walker to prevent falls and range of motion exercise with continuous passive motion and muscle strengthening.

Patients were instructed to complete a questionnaire to rate their pain level at rest and $45^{\circ}$ of knee flexion using the NRS. NRS assessments were performed four times daily until postoperative day 3 and once daily between postoperative day 4 and 7 . The results from these assessments were retrospectively analyzed to determine pain control. In addition, the number of IV-PCA attempts, total volume of infused PCA, and total volume of any additional analgesic (tramadol hydrochloride) infused were retrospectively analyzed. We also investigated complications associated with each pain control technique. To evaluate ankle dorsiflexion motor power, manual muscle testing (MMT) was performed. MedCalc ver. 15.2.2 (MedCalc Inc., Mariakerke, Belgium) was used to analyze the data collected. Statistical analysis to compare the difference among groups was performed using independent samples $t$-test and chi-square test. p-values less than 0.05 with $95 \%$ confidence interval were considered statistically significant.

Table 1. Demographic and Perioperative Data

\begin{tabular}{lccc}
\hline \multicolumn{1}{c}{ Characteristic } & $\begin{array}{c}\text { A group } \\
(\mathrm{ACB})\end{array}$ & $\begin{array}{c}\text { B group } \\
(\mathrm{ACB}+\mathrm{PSNB})\end{array}$ & p-value \\
\hline No. of patients & 100 & 100 & \\
Gender (male/female) & $12 / 88$ & $8 / 92$ & 0.915 \\
Age (yr) & $70.3 \pm 6.5$ & $71.2 \pm 6.9$ & 0.343 \\
Height (cm) & $150.6 \pm 7.6$ & $149.5 \pm 6.2$ & 0.263 \\
Weight (kg) & $63.4 \pm 9.7$ & $61.2 \pm 8.3$ & 0.086 \\
Preoperative NRS & $7.2 \pm 1.8$ & $7.6 \pm 2.0$ & 0.138 \\
MMT (ankle dorsiflexion) & $4.91 \pm 0.28$ & $4.92 \pm 0.27$ & 0.801 \\
Time to analgesia (min) & $10.7 \pm 4.3$ & $15.5 \pm 7.3$ & $<0.001$ \\
\hline
\end{tabular}

Values are presented as mean \pm standard deviation.

ACB: adductor canal block, PSNB: popliteal sciatic nerve block, NRS: numeric rating scale, MMT: manual muscle test. 


\section{Results}

There was no notable difference in age, gender, height, weight, and preoperative knee score between the groups (Table 1). Although group B had a significantly longer anesthesia time than group $\mathrm{A}$, the finding was not considered clinically significant.

Postoperative pain assessed using the NRS showed better results in group B than in group A on postoperative day 1 . The mean NRS pain scores during $45^{\circ}$ of knee flexion were significantly lower in group B than group $\mathrm{A}$ in the four tests performed on postoperative day $1(\mathrm{p}<0.001, \mathrm{p}=0.002, \mathrm{p}<0.001$, and $\mathrm{p}=0.012)$. Since then, despite there were no significant differences in NRS scores between the two groups, group B had lower NRS scores than group A except in the fourth test on postoperative day 2. Af-

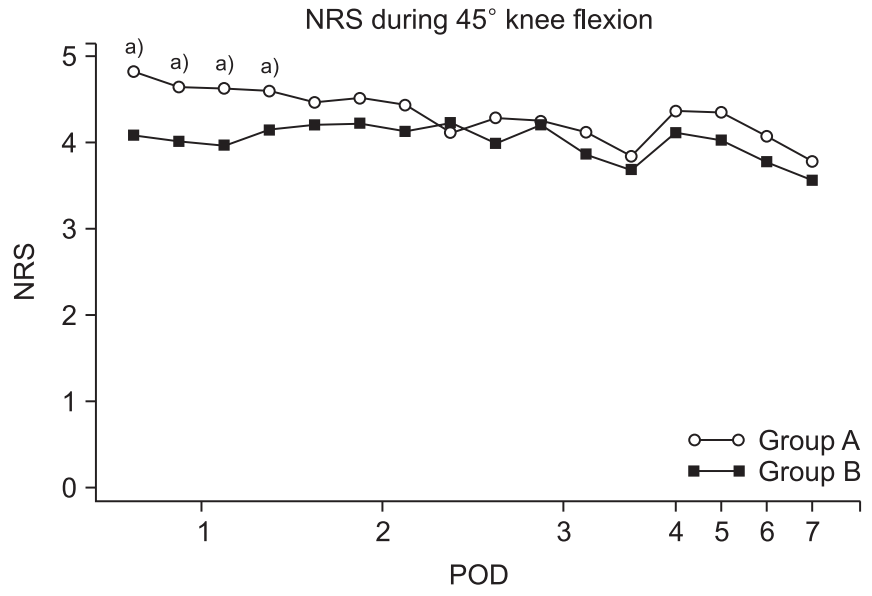

Fig. 1. Mean numeric rating scale (NRS) pain score during $45^{\circ}$ flexion of knee. POD: postoperative day.

${ }^{a)} \mathrm{p}$-values by independent-samples $t$-test; statistically significant $(\mathrm{p}<0.05)$.

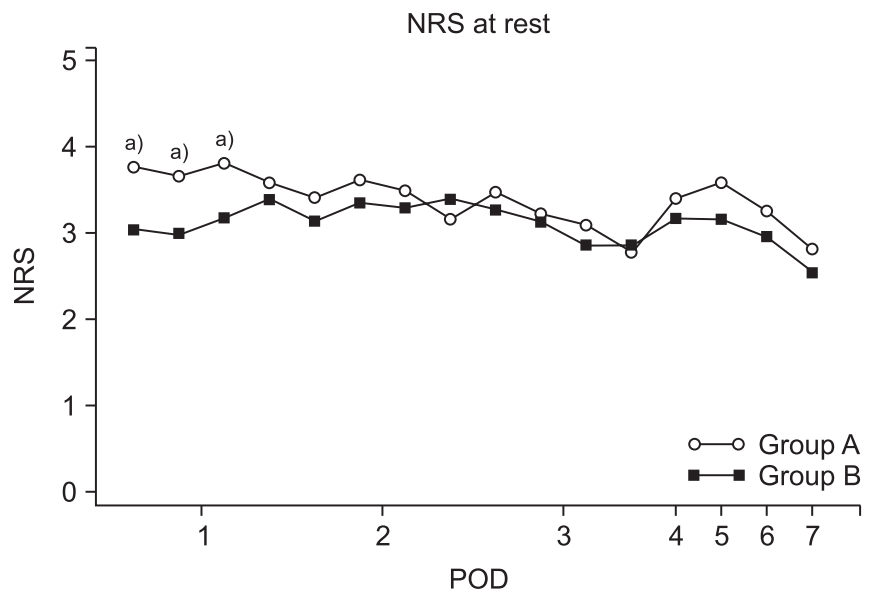

Fig. 2. Mean numeric rating scale (NRS) pain score at rest. POD: postoperative day.

${ }^{a)} \mathrm{p}$-values by independent-samples $t$-test; statistically significant $(\mathrm{p}<0.05)$. ter removal of the catheter for ACB, NRS scores increased slightly in both groups (Fig. 1).

The mean NRS scores at rest were significantly lower in group $\mathrm{B}$ than group A in three tests on postoperative day $1(\mathrm{p}<0.001$, $\mathrm{p}<0.001$, and $\mathrm{p}=0.001)$. Since then, despite there were no significant differences between two groups, group B had lower NRS scores than group A except in the fourth tests on postoperative day 2 and 3. After removal of the catheter for ACB, NRS scores increased slightly in both groups (Fig. 2).

On postoperative day 1 , group $B$ had a significantly lower number of IV-PCA attempts ( $\mathrm{p}=0.04$ ) (Fig. 3). On the other days, group $B$ had lower numbers of IV-PCA attempts without significant differences ( $\mathrm{p}=0.295$ and $\mathrm{p}=0.358$ ). Group B had a significantly lower total volume of PCA infused than group A ( $\mathrm{p}<0.001)$ (Fig. 4). So, group B had a significantly lower opioid consumption than group A (mean \pm standard deviation, $233.8 \pm 135.3 \mathrm{mcg}$

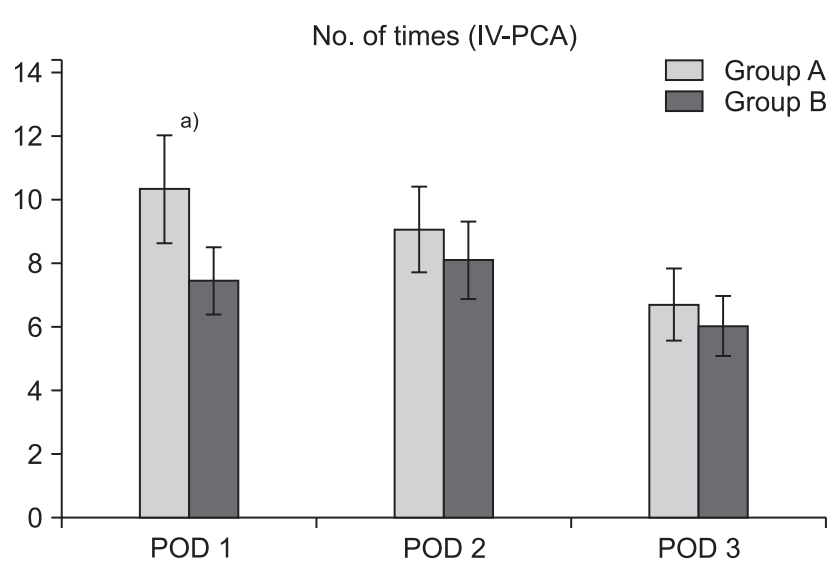

Fig. 3. Comparison of the mean number of times that patients pushed intravenous patient controlled analgesia (IV-PCA) button between groups. POD: postoperative day.

${ }^{a)} \mathrm{p}$-values by independent-samples $t$-test; statistically significant $(\mathrm{p}<0.05)$.

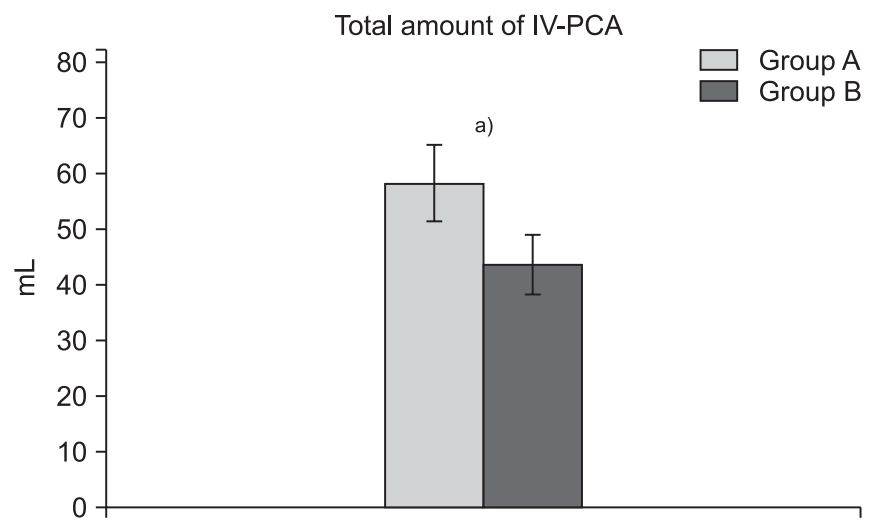

Fig. 4. Comparison of total intravenous patient controlled analgesia (IVPCA) amounts between the two groups.

${ }^{a)} \mathrm{p}$-values by independent-samples $t$-test; statistically significant $(\mathrm{p}<0.05)$. 
and $174.7 \pm 107.2 \mathrm{mcg}$, respectively; $\mathrm{p}=0.024)$.

However, no significant difference in the total volume of additional analgesic agents and PCA-related complications was found between groups. Catheter insertion for continuous ACB failed to be maintained in 18 cases (9\%) in both groups. The catheter was immediately replaced in each case as soon as it was found withdrawn. Post-ACB saphenous neuropathy was identified in 12 cases (6\%); all patients fully recovered within three weeks. The incidence of nausea and vomiting during IV-PCA was not significantly different between groups. Thirty-five (35\%) patients in group B developed foot drop immediately after surgery; they all fully recovered on postoperative day 1 . Postoperative ankle dorsiflexion motor power assessed by MMT was not significantly different between groups ( $\mathrm{p}=0.801$ ); however, ankle dorsiflexion motor power on postoperative day 1 was significantly lower in group B than group A (mean \pm standard deviation, $4.2 \pm 0.7$ and $2.1 \pm 1.6$, respectively; $\mathrm{p}<0.001$ ). On postoperative day 2 , most patients recovered from foot drop, so there were no significant differences in terms of ankle dorsiflexion motor power between two groups (mean \pm standard deviation, $4.6 \pm 0.5$ vs. $4.54 \pm 0.5 ; \mathrm{p}=0.09$ ).

\section{Discussion}

In this study, we sought to determine the effectiveness of PSNB in managing persistent postoperative posterior knee pain despite ACB. We found that the combination of ACB and PSNB was more effective than ACB alone in the management of persistent postoperative pain. Patients in group $B$ who received ACB and PSNB had significantly lower NRS scores at rest and $45^{\circ}$ of knee flexion on postoperative day 1 than those in group A who received ACB alone. In addition, the number of IV-PCA attempts on postoperative day 1 and the total volume of PCA infused were significantly lower in group B than group A. Thus, significantly lower NRS scores at rest and $45^{\circ}$ of knee flexion as well as less analgesic use through PCA and lower opioid consumption demonstrate that continuous ACB combined with PSNB was more effective than continuous $\mathrm{ACB}$ alone in controlling pain in the early postoperative period. Similarly, two meta-analyses, albeit not about the ACB, concluded that SNB in addition to FNB can significantly diminish early postoperative knee pain and reduce opioid consumption compared with FBN alone for patients undergoing $\mathrm{TKA}^{6,12)}$. Reduced persistent postoperative pain following PSNB appears to have occurred mainly in the posterior aspect of the knee. Pain relieving effects also seem to have affected the lateral or anterolateral sides of the knee given that the common peroneal nerve, a branch of the sciatic nerve, provides some of its articular branches to this area.

However, the effects of single-shot PSNB usually disappeared on postoperative day 2 . We found that NRS scores at rest and $45^{\circ}$ of knee flexion showed no significant difference between groups on postoperative day 2 and later. On postoperative day 4, after the removal of catheters used for continuous $\mathrm{ACB}$, both groups showed slightly increased NRS scores at rest and $45^{\circ}$ of knee flexion. This outcome is seen as a rebound phenomenon, which was initially reported in a previous study investigating the efficacy of periarticular multimodal drug injections into the area surrounding the joint space after TKA ${ }^{13)}$.

The major sensory nerves of the knee include the femoral, obturator, lateral femoral cutaneous nerves in addition to the sciatic nerve. Until now, peripheral nerve block techniques developed for the management of postoperative pain after TKA have focused on the femoral nerve, which is linked to pain in the anterior and medial aspects of the knee ${ }^{2}$. However, there have been studies reporting that FNB alone cannot provide sufficient pain relief ${ }^{14,15)}$. Weber et al. ${ }^{15)}$ reported that $67 \%$ of patients who underwent FNB required additional PSNB. Chelly et al. ${ }^{16)}$ and Eledjam et al. ${ }^{17)}$ demonstrated the excellent efficacy of continuous FNB combined with a single-shot SNB. While the efficacy of FNB followed by additional SNB has been reported in previous studies, there has been no study addressing the efficacy of ACB combined with a SNB in Korea and abroad. Therefore, the significance of this study lies in the fact that we attempted to investigate the effects of $A C B$, which is known to be as effective as $\mathrm{FNB}$ in reducing pain and preventing deterioration in quadriceps muscle function ${ }^{9,10)}$, in combination with SNB.

The complications resulting from continuous ACB included catheter failure and neuropathy. The catheter failure rate was 9\% (18/200) during continuous ACB. Postoperative failure to maintain the catheter was identified in patients showing no pain relief even after drug administration by running a cotton soaked with alcohol on the superior medial aspects of the ankle where the saphenous nerve ends to compare cold sensation on the opposite side. Once the catheter failure was identified, ACB was immediately performed again. We found a success rate of $91 \%$ for ACB, which is similar to the rate reported in previous studies $^{18,19)}$. The catheter was placed in the middle of the thigh and positioned under the tourniquet, which made it easy to be moved during surgery. Furthermore, the catheter was inserted through the sartorius muscle so that it could be pulled out of the adductor canal during movements in early rehabilitation or walking. Neuropathy occurred in 12/200 (6\%) of all patients. Major symptoms reported by these patients included electric shock sensation at 
the area innervated by the saphenous nerve and paresthesia. The causes of nerve damage following peripheral nerve blocks include direct injury, intraneural injection of anesthetic, hematoma formation, blood flow reduction, and infection. Considering the ultrasound-guided catheter placement and use of nerve block, we think it was more likely that neuropathy in our patients resulted from reduced blood flow due to the use of tourniquet than direct injury or intraneural injection. Most patients fully recovered from neuropathic symptoms within 3 weeks without medication or any other treatment. The reports of complications associated with SNBs are still lacking because SNB is not commonly performed compared to other peripheral nerve blocks. We found no incidence of neuropathy after SNBs. However, among the 100 patients who received SNB, 35 (35\%) experienced foot drop, which is lower than the incidence of foot drop (range, 65\% to $68 \%$ ) reported in other studies of patients undergoing $\mathrm{SNBs}^{14,20)}$. Some surgeons suggest SNB should not be performed for anxiety of masking a surgical injury to the peroneal nerve ${ }^{21)}$ and risk of fall. But the incidence of peroneal nerve injury based on more recent observations is estimated to be $0.79 \%{ }^{22)}$. In addition, patients with foot drop were told in advance it is a common complication after nerve block and advised to take extra caution to prevent fall. These patients recovered from foot drop within 24 hours of TKA. So, we believe that foot drop after PSNB does not have clinically significant impact.

Our study had several limitations. First, it was a retrospective, nonrandomized study. Differences in patient characteristics and surgical and nerve block techniques between the groups may have affected the measured outcomes. To minimize the influence of confounding factors, we selected patients with similar baseline characteristics. Moreover, TKAs were performed by the same senior surgeon, and peripheral nerve blocks by the same anesthesiologist using standard protocols. Second, we used 20 $\mathrm{mL}$ ropivacaine for the PSNB; however, it is not clear whether the amount of drugs we used was adequate for PSNB. Further studies are necessary to determine the appropriate amount of analgesics that can maximize pain relieving effects and reduce the incidence of foot drop. Finally, in addition to peripheral nerve blocks, an aggressive postoperative multimodal analgesic regimen (IV-PCA and oral analgesics) was used in both groups because of ethical reasons. It is likely to have contributed to the low pain scores. Therefore, the study may have been underpowered to prove the combined effect of PSNB and ACB for pain control after TKA.

\section{Conclusions}

This study suggests that PSNB is highly effective in reducing opioid consumption and severe early pain after TKA even after continuous ACB. However, because foot drop occurred in a number of patients, particular care must be taken to prevent fall injuries. Further research is needed to determine the amount of drugs and technique for reducing the incidence of foot drop.

\section{Conflict of Interest}

No potential conflict of interest relevant to this article was reported.

\section{References}

1. Singelyn FJ, Ferrant T, Malisse MF, Joris D. Effects of intravenous patient-controlled analgesia with morphine, continuous epidural analgesia, and continuous femoral nerve sheath block on rehabilitation after unilateral total-hip arthroplasty. Reg Anesth Pain Med. 2005;30:452-7.

2. Paul JE, Arya A, Hurlburt L, Cheng J, Thabane L, Tidy A, Murthy Y. Femoral nerve block improves analgesia outcomes after total knee arthroplasty: a meta-analysis of randomized controlled trials. Anesthesiology. 2010;113:114462.

3. Ilfeld BM, Le LT, Meyer RS, Mariano ER, Vandenborne K, Duncan PW, Sessler DI, Enneking FK, Shuster JJ, Theriaque DW, Berry LF, Spadoni EH, Gearen PF. Ambulatory continuous femoral nerve blocks decrease time to discharge readiness after tricompartment total knee arthroplasty: a randomized, triple-masked, placebo-controlled study. Anesthesiology. 2008;108:703-13.

4. Safa B, Gollish J, Haslam L, McCartney CJ. Comparing the effects of single shot sciatic nerve block versus posterior capsule local anesthetic infiltration on analgesia and functional outcome after total knee arthroplasty: a prospective, randomized, double-blinded, controlled trial. J Arthroplasty. 2014;29:1149-53.

5. Abdallah FW, Brull R. Is sciatic nerve block advantageous when combined with femoral nerve block for postoperative analgesia following total knee arthroplasty? A systematic review. Reg Anesth Pain Med. 2011;36:493-8.

6. Abdallah FW, Madjdpour C, Brull R. Is sciatic nerve block advantageous when combined with femoral nerve block for postoperative analgesia following total knee arthroplasty? a 
meta-analysis. Can J Anaesth. 2016;63:552-68.

7. Li J, Deng X, Jiang T. Combined femoral and sciatic nerve block versus femoral and local infiltration anesthesia for pain control after total knee arthroplasty: a meta-analysis of randomized controlled trials. J Orthop Surg Res. 2016;11:158.

8. Wang C, Cai XZ, Yan SG. Comparison of periarticular multimodal drug injection and femoral nerve block for postoperative pain management in total knee arthroplasty: a systematic review and meta-analysis. J Arthroplasty. 2015; 30:1281-6.

9. Gao F, Ma J, Sun W, Guo W, Li Z, Wang W. Adductor canal block versus femoral nerve block for analgesia after total knee arthroplasty: a systematic review and meta-analysis. Clin J Pain. 2017;33:356-368.

10. Koh IJ, Choi YJ, Kim MS, Koh HJ, Kang MS, In Y. Femoral nerve block versus adductor canal block for analgesia after total knee arthroplasty. Knee Surg Relat Res. 2017;29:87-95.

11. Sinha A, Chan VW. Ultrasound imaging for popliteal sciatic nerve block. Reg Anesth Pain Med. 2004;29:130-4.

12. Grape S, Kirkham KR, Baeriswyl M, Albrecht E. The analgesic efficacy of sciatic nerve block in addition to femoral nerve block in patients undergoing total knee arthroplasty: a systematic review and meta-analysis. Anaesthesia. 2016;71: 1198-209.

13. Mullaji A, Kanna R, Shetty GM, Chavda V, Singh DP. Efficacy of periarticular injection of bupivacaine, fentanyl, and methylprednisolone in total knee arthroplasty:a prospective, randomized trial. J Arthroplasty. 2010;25:851-7.

14. Sinha SK, Abrams JH, Arumugam S, D’Alessio J, Freitas DG, Barnett JT, Weller RS. Femoral nerve block with selective tibial nerve block provides effective analgesia without foot drop after total knee arthroplasty: a prospective, randomized, observer-blinded study. Anesth Analg. 2012;115:202-6.

15. Weber A, Fournier R, Van Gessel E, Gamulin Z. Sciatic nerve block and the improvement of femoral nerve block analgesia after total knee replacement. Eur J Anaesthesiol. 2002;19:834-6.

16. Chelly JE, Greger J, Gebhard R, Coupe K, Clyburn TA, Buckle R, Criswell A. Continuous femoral blocks improve recovery and outcome of patients undergoing total knee arthroplasty. J Arthroplasty. 2001;16:436-45.

17. Eledjam JJ, Cuvillon P, Capdevila X, Macaire P, Serri S, Gaertner E, Jochum D; French study group. Postoperative analgesia by femoral nerve block with ropivacaine $0.2 \%$ after major knee surgery: continuous versus patient-controlled techniques. Reg Anesth Pain Med. 2002;27:604-11.

18. Mahadevan D, Walter RP, Minto G, Gale TC, McAllen CJ, Oldman M. Combined femoral and sciatic nerve block vs combined femoral and periarticular infiltration in total knee arthroplasty: a randomized controlled trial. J Arthroplasty. 2012;27:1806-11.

19. Singelyn FJ, Deyaert M, Joris D, Pendeville E, Gouverneur JM. Effects of intravenous patient-controlled analgesia with morphine, continuous epidural analgesia, and continuous three-in-one block on postoperative pain and knee rehabilitation after unilateral total knee arthroplasty. Anesth Analg. 1998;87:88-92.

20. Danelli G, Fanelli A, Ghisi D, Moschini E, Rossi M, Ortu A, Baciarello M, Fanelli G. Ultrasound vs nerve stimulation multiple injection technique for posterior popliteal sciatic nerve block. Anaesthesia. 2009;64:638-42.

21. Nercessian OA, Ugwonali OF, Park S. Peroneal nerve palsy after total knee arthroplasty. J Arthroplasty. 2005;20:1068-73.

22. Jacob AK, Mantilla CB, Sviggum HP, Schroeder DR, Pagnano MW, Hebl JR. Perioperative nerve injury after total knee arthroplasty: regional anesthesia risk during a 20-year cohort study. Anesthesiology. 2011;114:311-7. 